\title{
Fulfilling the promise of Mendelian randomization
}

\author{
Joseph K. Pickrell ${ }^{\dagger 1,2}$ \\ ${ }^{1}$ New York Genome Center, New York, NY \\ 2 Department of Biological Sciences, Columbia University, New York, NY \\ $\dagger$ Correspondence to: jkpickrell@nygenome.org
}

April 15, 2015 


\section{Introduction}

Many important questions in medicine involve questions about causality. For example, do low levels of high-density lipoproteins (HDL) cause heart disease? Does high body mass index (BMI) cause type 2 diabetes? Or are these traits simply correlated in the population for other reasons? The gold standard study design for answering this type of question is the randomized controlled trial, which requires identifying an intervention that influences the potential causal factor (for example a drug), randomly assigning individuals to receive the intervention, and monitoring outcomes. In this study design, the use of randomization allows one to isolate the causal effect of a single variable (e.g. the drug) from the effects of potential confounding variables. However, randomized controlled trials are sometimes impractical (for example, no intervention may exist), expensive, or indeed unethical (for a tongue-in-cheek example, see Smith and Pell [31]).

In the absence of a randomized trial, some hints about the causal relationship between traits can come from observational epidemiology. If two variables are correlated in the population (e.g. low HDL cholesterol is correlated with higher risk of heart disease [14]) then this might indicate a relationship worth examining further. On the other hand, observational correlations are potentially confounded by a large number of factors (e.g. Taubes [34] and references therein), and following up non-causal relationships with randomized controlled trials leads to large investments of time and money with little payoff. Classic examples of this problem include trials of hormone replacement therapy in cardiovascular disease [29] or beta-carotene in lung cancer [36].

A useful attack on the problem of confounding in observational epidemiology comes from genetics, and is now called "Mendelian randomization" (the method is generally attributed to Katan [19], but see Katan [20]). The intuition is as follows: if two traits are causally related, a genetic variant that influences the first trait (for example, BMI) is expected to have a "knock-on" effect on the second trait (for example, type 2 diabetes), while this is not the case if the two traits are correlated for other reasons. Since genetic variants are inherited at conception and (to a first approximation) do not change over the course of an individual's lifetime, the random inheritance of a genetic variant acts like the randomization in a clinical trial to help avoid the effects of confounding. In the simplest form, Mendelian randomization thus simply involves testing to see if a genetic variant has effects on two traits [19]. More elaborate methods allow one to estimate the magnitude of the causal effect of one trait on the other, and to include multiple genetic variants; for more in-depth description of the statistical methodology, see Didelez and Sheehan [11], VanderWeele et al. [39] and Davey Smith and Hemani [9].

The Mendelian randomization approach is now extremely popular (e.g. [4, 5, 10, 13, 16, 17, 22, 24, 27, 41, 42]). For example, this approach has recently been used to argue that alcohol consumption increases risk of heart disease [16], and that increasing serum iron levels decreases risk of Parkinson's disease [27]. These findings have potentially important public health implications.

However, it is well-known that causal inference using Mendelian randomization requires strong assumptions about the physiological mechanisms by which genetic variants exert their effects ([9, 39]. Specifically, if a genetic variant influences two phenotypes through different mechanisms, then Mendelian randomization will give misleading results. For example, a genetic variant in the gene LGR4 influences both bone mineral density and risk of gallbladder cancer [33], but it seems implausible to suggest that low bone mineral density causes cancer. Instead, it seems more likely that this genetic variant alters some molecular pathway (or multiple pathways) that is important for both phenotypes. Here, we describe two pieces of evidence that make us moderately skeptical of most of the causal claims made to date using Mendelian randomization, and suggest some ways forward. 


\section{Two reasons for skepticism}

The first reason for skepticism is that in the nearly 30 years of Mendelian randomization, arguably no new causal relationship has been identified with this approach and subsequently verified in a randomized controlled trial. We assembled a list of 29 applications of Mendelian randomization from two recent reviews [3, 9] (Supplementary Table 1). Of these 29 pairs of traits, we identified five that were also targets of randomized controlled trials. In nearly all of these case, the Mendelian randomization study was performed after the randomized controlled trial, and generally confirmed the results of the trial (Table 11). For example, Mendelian randomization studies have provided additional evidence that BMI causally influences atherosclerosis [21], and that HDL levels do not appear to causally influence risk of cardiovascular disease [41]. These studies have provided important, independent evidence about the relationships between these traits, but it seems the most rigorous test of the Mendelian randomization methodology would be to identify a new causal relationship and then to subsequently confirm it with a controlled trial.

The one case where Mendelian randomization provided support for a causal relationship prior to the publication of a randomized controlled trial was the case of homocysteine levels and stroke [5]. This study inferred that increased homocysteine levels cause higher stroke risk. However, randomized controlled trials of folic acid (which reduces levels of circulating homocysteine) have produced conflicting results [18, 32]. There are a number of potential explanations for this (indeed, the robustness of the Mendelian randomization study has been questioned [23]), but overall the track record of Mendelian randomization is sparse.

The second reason for skepticism is more fundamental. Specifically, one of the core assumptions when using Mendelian randomization to infer causality is that the genetic variants used in the study have a direct effect on one trait (the "causal" trait), but only an indirect effect on the other (the "caused" trait). That is, the genetic variants used in the study are assumed to have no influence on confounding factors that influences both traits. This is often referred to as an assumption of no "pleiotropy" [9]. However, it is difficult to know a priori whether this assumption is valid, and recent work in human genetics has shown that genetic variants often have effects on different traits. For example, genetic variants that influence one autoimmune disease also often influence others [6], and variants that influence one lipid trait also often influence other lipid traits [35]. This is not limited to traits that are intuitively related, however-large genome-wide association studies are finding that genetic variants often influence many aspects of physiology [2]. For example, genetic variants that influence HDL cholesterol levels have correlated effects on whether an individual went to college [2]; a naive interpretation of this might suggest the (rather nonsensical) conclusion that HDL-raising drugs should increase education levels.

It is sometimes suggested that using a large number of genetic variants in Mendelian randomization (combined into a single score) offers a way to partially avoid this problem (e.g. [9, 17]). To test this, we performed simulations of such an approach in a situation where two traits are not in fact causally related (Figure 1A, see Supplementary Information for details). We found that even a small number of pleiotropic loci (those that influence a confounding variable) leads to false positives, and that this problem grows worse as sample sizes increase (Figure 1B). In empirical data, this effect has been seen by Evans et al. [12], who noted that a related approach identified several apparently spurious causal relationships between traits. Before making a strong causal conclusion from a Mendelian randomization study, then, it seems necessary to have a good molecular and physiological understanding of the effects of all the genetic variants used in the study. For many traits of interest, our understanding of biology is not strong enough for this to be reasonable.

\section{Fulfilling the promise of Mendelian randomization}

The principle of Mendelian randomization, however, remains tremendously promising. What are the ways forward? One possibility is to treat negative results from Mendelian randomization with more confidence 
than positive results-if two traits are correlated in the population but a Mendelian randomization study suggests they are not causally related, this is evidence that the population-level correlation is driven by some unobserved confounder [39]. Another approach might be to focus on developing a detailed molecular understanding of variants in an individual gene and their downstream physiological effects; this approach is particularly useful if the gene is a potential drug target [28]. Finally, another possibility comes from the ongoing development of new statistical methods like bi-directional Mendelian randomization (e.g. [37, 40]), which use many genetic variants to increase the robustness of causal inference. These methods are currently in early stages of development and evaluation. Until they reach maturity, reports of causal relationships identified through Mendelian randomization should be treated with the same skepticism as such claims made from observational epidemiology. 
Table 1: List of causal relationships evaluated by Mendelian randomization and randomized controlled trials, compiled from Table 1 of Davey Smith and Hemani [9] and Table 1 of Burgess and Thompson [3]. For the complete list of studies, see Supplementary Table 1 . MR = Mendelian randomization, RCT = randomized controlled trial.

\begin{tabular}{|c|c|c|c|c|c|c|}
\hline Trait & Outcome & MR effect & Reference & RCT effect & Reference & Performed first \\
\hline cholesterol levels & cancer & None & {$[38]$} & None & {$[7]$} & RCT \\
HDL cholesterol & myocardial infarction & None & {$[41]$} & None & {$[1]$} & RCT \\
homocysteine & stroke & + & {$[5]$} & Conflicting & {$[18,32]$} & MR \\
BMI & cartoid thickness & + & {$[21]$} & + & {$[15,30]$} & RCT \\
interuterine folate & neural tube defects & - & {$[8]$} & - & {$[25]$} & RCT \\
\hline
\end{tabular}



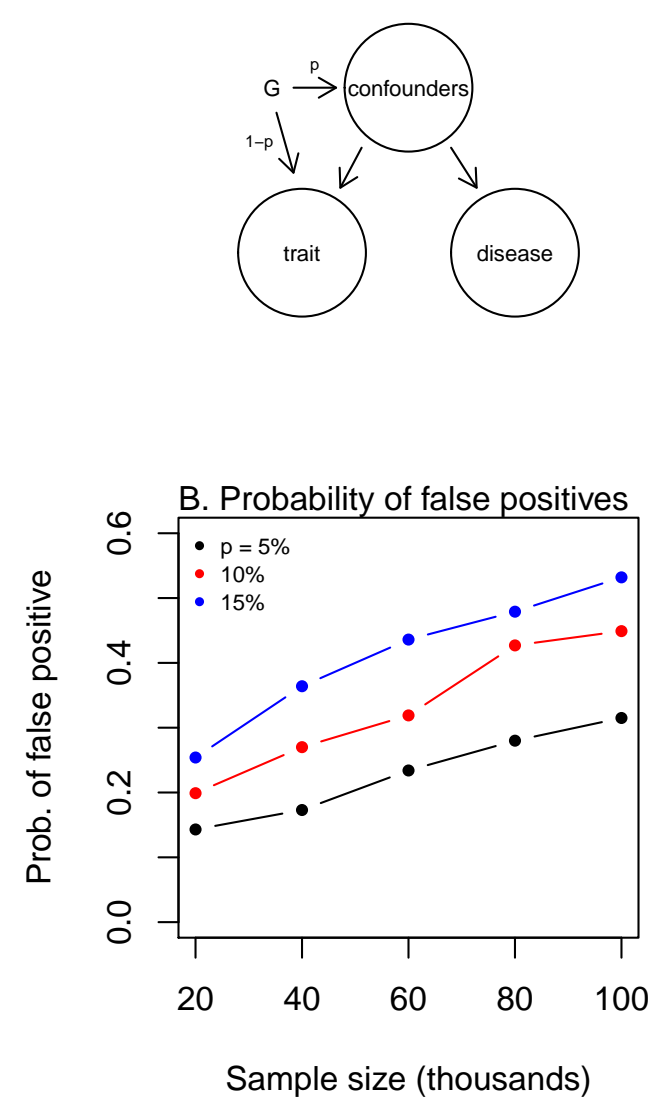

Figure 1. Simulations of Mendelian randomization in the absence of a causal relationship between a trait and a disease. A. Simulated scenario. For each simulation, we simulated 100 genetic variants $(G)$, which either 1) influence a trait or 2) influence both the trait and the disease, with proportions $1-p$ and $p$, respectively. Arrows represent causal relationships [26]. The confounders are not directly simulated; see Supplementary Material for details. B. Probability of incorrectly inferring a causal relationship between a trait and a disease in this situation. We simulated Mendelian randomization studies with different sample sizes and values of $p$, and in each simulation tested for a causal relationship between the trait and the disease. Each point shows the proportion of 1,000 independent simulations where the causal effect of the trait on the disease was "significant" as judged by $P<0.05$. In all simulations, we assumed that the effects of a variant on the trait and on the disease are uncorrelated; see Figure S1 for simulations where these effect sizes are correlated. 


\section{Acknowledgements.}

We thank Molly Przeworski for helpful comments on an earlier draft of this manuscript.

\section{References}

[1] Barter, P. J., Caulfield, M., Eriksson, M., Grundy, S. M., Kastelein, J. J. P., Komajda, M., Lopez-Sendon, J., Mosca, L., Tardif, J.-C., Waters, D. D., et al., 2007. Effects of torcetrapib in patients at high risk for coronary events. N Engl J Med, 357(21):2109-22.

[2] Bulik-Sullivan, B., Finucane, H. K., Anttila, V., Gusev, A., Day, F. R., Perry, J. R., Patterson, N., Robinson, E., Daly, M. J., Price, A. L., et al., 2015. An Atlas of Genetic Correlations across Human Diseases and Traits. bioRxiv, :014498.

[3] Burgess, S. and Thompson, S. G., 2015. Mendelian Randomization: Methods for using Genetic Variants in Causal Estimation. CRC Press.

[4] C Reactive Protein Coronary Heart Disease Genetics Collaboration (CCGC), Wensley, F., Gao, P., Burgess, S., Kaptoge, S., Di Angelantonio, E., Shah, T., Engert, J. C., Clarke, R., Davey-Smith, G., et al., 2011. Association between $\mathrm{C}$ reactive protein and coronary heart disease: mendelian randomisation analysis based on individual participant data. BMJ, 342:d548.

[5] Casas, J. P., Bautista, L. E., Smeeth, L., Sharma, P., and Hingorani, A. D., 2005. Homocysteine and stroke: evidence on a causal link from mendelian randomisation. Lancet, 365(9455):224-32.

[6] Cotsapas, C., Voight, B. F., Rossin, E., Lage, K., Neale, B. M., Wallace, C., Abecasis, G. R., Barrett, J. C., Behrens, T., Cho, J., et al., 2011. Pervasive sharing of genetic effects in autoimmune disease. PLoS Genet, 7(8):e1002254.

[7] Dale, K. M., Coleman, C. I., Henyan, N. N., Kluger, J., and White, C. M., 2006. Statins and cancer risk: a meta-analysis. JAMA, 295(1):74-80.

[8] Davey Smith, G. and Ebrahim, S., 2003. 'Mendelian randomization': can genetic epidemiology contribute to understanding environmental determinants of disease? Int J Epidemiol, 32(1):1-22.

[9] Davey Smith, G. and Hemani, G., 2014. Mendelian randomization: genetic anchors for causal inference in epidemiological studies. Hum Mol Genet, 23(R1):R89-98.

[10] De Silva, N. M. G., Freathy, R. M., Palmer, T. M., Donnelly, L. A., Luan, J., Gaunt, T., Langenberg, C., Weedon, M. N., Shields, B., Knight, B. A., et al., 2011. Mendelian randomization studies do not support a role for raised circulating triglyceride levels influencing type 2 diabetes, glucose levels, or insulin resistance. Diabetes, 60(3):1008-18.

[11] Didelez, V. and Sheehan, N., 2007. Mendelian randomization as an instrumental variable approach to causal inference. Stat Methods Med Res, 16(4):309-30.

[12] Evans, D. M., Brion, M. J. A., Paternoster, L., Kemp, J. P., McMahon, G., Munafò, M., Whitfield, J. B., Medland, S. E., Montgomery, G. W., GIANT Consortium, et al., 2013. Mining the human phenome using allelic scores that index biological intermediates. PLoS Genet, 9(10):e1003919.

[13] Fall, T., Hägg, S., Mägi, R., Ploner, A., Fischer, K., Horikoshi, M., Sarin, A.-P., Thorleifsson, G., Ladenvall, C., Kals, M., et al., 2013. The role of adiposity in cardiometabolic traits: a Mendelian randomization analysis. PLoS Med, 10(6):e1001474. 
[14] Gordon, T., Castelli, W. P., Hjortland, M. C., Kannel, W. B., and Dawber, T. R., 1977. High density lipoprotein as a protective factor against coronary heart disease. The Framingham Study. Am J Med, 62(5):707-14.

[15] Hjerkinn, E. M., Abdelnoor, M., Breivik, L., Bergengen, L., Ellingsen, I., Seljeflot, I., Aase, O., Ole Klemsdal, T., Hjermann, I., and Arnesen, H., et al., 2006. Effect of diet or very long chain omega-3 fatty acids on progression of atherosclerosis, evaluated by carotid plaques, intima-media thickness and by pulse wave propagation in elderly men with hypercholesterolaemia. Eur J Cardiovasc Prev Rehabil, 13(3):325-33.

[16] Holmes, M. V., Dale, C. E., Zuccolo, L., Silverwood, R. J., Guo, Y., Ye, Z., Prieto-Merino, D., Dehghan, A., Trompet, S., Wong, A., et al., 2014a. Association between alcohol and cardiovascular disease: Mendelian randomisation analysis based on individual participant data. BMJ, 349:g4164.

[17] Holmes, M. V., Lange, L. A., Palmer, T., Lanktree, M. B., North, K. E., Almoguera, B., Buxbaum, S., Chandrupatla, H. R., Elbers, C. C., Guo, Y., et al., 2014b. Causal effects of body mass index on cardiometabolic traits and events: a Mendelian randomization analysis. Am J Hum Genet, 94(2):198208.

[18] Huo, Y., Li, J., Qin, X., Huang, Y., Wang, X., Gottesman, R. F., Tang, G., Wang, B., Chen, D., He, M., et al., 2015. Efficacy of Folic Acid Therapy in Primary Prevention of Stroke Among Adults With Hypertension in China: The CSPPT Randomized Clinical Trial. JAMA, .

[19] Katan, M. B., 1986. Apolipoprotein E isoforms, serum cholesterol, and cancer. Lancet, 1(8479):507-8.

[20] Katan, M. B., 2004. Commentary: Mendelian Randomization, 18 years on. Int J Epidemiol, 33(1):101.

[21] Kivimäki, M., Smith, G. D., Timpson, N. J., Lawlor, D. A., Batty, G. D., Kähönen, M., Juonala, M., Rönnemaa, T., Viikari, J. S. A., Lehtimäki, T., et al., 2008. Lifetime body mass index and later atherosclerosis risk in young adults: examining causal links using Mendelian randomization in the Cardiovascular Risk in Young Finns study. Eur Heart J, 29(20):2552-60.

[22] Klerk, M., Verhoef, P., Clarke, R., Blom, H. J., Kok, F. J., Schouten, E. G., and MTHFR Studies Collaboration Group, 2002. MTHFR $677 \mathrm{C}->\mathrm{T}$ polymorphism and risk of coronary heart disease: a meta-analysis. JAMA, 288(16):2023-31.

[23] Lewis, S. J., Ebrahim, S., and Davey Smith, G., 2005. Meta-analysis of MTHFR 677C-¿T polymorphism and coronary heart disease: does totality of evidence support causal role for homocysteine and preventive potential of folate? BMJ, 331(7524):1053.

[24] Lim, E. T., Würtz, P., Havulinna, A. S., Palta, P., Tukiainen, T., Rehnström, K., Esko, T., Mägi, R., Inouye, M., Lappalainen, T., et al., 2014. Distribution and Medical Impact of Loss-of-Function Variants in the Finnish Founder Population. PLoS Genet, 10(7):e1004494.

[25] MRC Vitamin Study Research Group et al., 1991. Prevention of neural tube defects: results of the Medical Research Council Vitamin Study. The lancet, 338(8760):131-137.

[26] Pearl, J., 2000. Causality: models, reasoning and inference, volume 29. Cambridge Univ Press.

[27] Pichler, I., Del Greco M, F., Gögele, M., Lill, C. M., Bertram, L., Do, C. B., Eriksson, N., Foroud, T., Myers, R. H., PD GWAS Consortium, et al., 2013. Serum iron levels and the risk of Parkinson disease: a Mendelian randomization study. PLoS Med, 10(6):e1001462. 
[28] Plenge, R. M., Scolnick, E. M., and Altshuler, D., 2013. Validating therapeutic targets through human genetics. Nat Rev Drug Discov, 12(8):581-94.

[29] Rossouw, J. E., Anderson, G. L., Prentice, R. L., LaCroix, A. Z., Kooperberg, C., Stefanick, M. L., Jackson, R. D., Beresford, S. A. A., Howard, B. V., Johnson, K. C., et al., 2002. Risks and benefits of estrogen plus progestin in healthy postmenopausal women: principal results From the Women's Health Initiative randomized controlled trial. JAMA, 288(3):321-33.

[30] Shai, I., Spence, J. D., Schwarzfuchs, D., Henkin, Y., Parraga, G., Rudich, A., Fenster, A., Mallett, C., Liel-Cohen, N., Tirosh, A., et al., 2010. Dietary intervention to reverse carotid atherosclerosis. Circulation, 121(10):1200-8.

[31] Smith, G. C. S. and Pell, J. P., 2003. Parachute use to prevent death and major trauma related to gravitational challenge: systematic review of randomised controlled trials. BMJ, 327(7429):1459-61.

[32] Study of the Effectiveness of Additional Reductions in Cholesterol and Homocysteine (SEARCH) Collaborative Group, Armitage, J. M., Bowman, L., Clarke, R. J., Wallendszus, K., Bulbulia, R., Rahimi, K., Haynes, R., Parish, S., Sleight, P., et al., 2010. Effects of homocysteine-lowering with folic acid plus vitamin B12 vs placebo on mortality and major morbidity in myocardial infarction survivors: a randomized trial. JAMA, 303(24):2486-94.

[33] Styrkarsdottir, U., Thorleifsson, G., Sulem, P., Gudbjartsson, D. F., Sigurdsson, A., Jonasdottir, A., Jonasdottir, A., Oddsson, A., Helgason, A., Magnusson, O. T., et al., 2013. Nonsense mutation in the LGR4 gene is associated with several human diseases and other traits. Nature, 497(7450):517-20.

[34] Taubes, G., 1995. Epidemiology faces its limits. Science, 269(5221):164-9.

[35] Teslovich, T. M., Musunuru, K., Smith, A. V., Edmondson, A. C., Stylianou, I. M., Koseki, M., Pirruccello, J. P., Ripatti, S., Chasman, D. I., Willer, C. J., et al., 2010. Biological, clinical and population relevance of 95 loci for blood lipids. Nature, 466(7307):707-713.

[36] The Alpha-Tocopherol Beta Carotene Cancer Prevention Study Group, 1994. The effect of vitamin $\mathrm{E}$ and beta carotene on the incidence of lung cancer and other cancers in male smokers. The AlphaTocopherol, Beta Carotene Cancer Prevention Study Group. N Engl J Med, 330(15):1029-35.

[37] Timpson, N. J., Nordestgaard, B. G., Harbord, R. M., Zacho, J., Frayling, T. M., Tybjærg-Hansen, A., and Smith, G. D., 2011. C-reactive protein levels and body mass index: elucidating direction of causation through reciprocal Mendelian randomization. Int J Obes (Lond), 35(2):300-8.

[38] Trompet, S., Jukema, J. W., Katan, M. B., Blauw, G. J., Sattar, N., Buckley, B., Caslake, M., Ford, I., Shepherd, J., Westendorp, R. G. J., et al., 2009. Apolipoprotein e genotype, plasma cholesterol, and cancer: a Mendelian randomization study. Am J Epidemiol, 170(11):1415-21.

[39] VanderWeele, T. J., Tchetgen Tchetgen, E. J., Cornelis, M., and Kraft, P., 2014. Methodological challenges in mendelian randomization. Epidemiology, 25(3):427-35.

[40] Vimaleswaran, K. S., Berry, D. J., Lu, C., Tikkanen, E., Pilz, S., Hiraki, L. T., Cooper, J. D., Dastani, Z., Li, R., Houston, D. K., et al., 2013. Causal relationship between obesity and vitamin D status: bi-directional Mendelian randomization analysis of multiple cohorts. PLoS Med, 10(2):e1001383.

[41] Voight, B. F., Peloso, G. M., Orho-Melander, M., Frikke-Schmidt, R., Barbalic, M., Jensen, M. K., Hindy, G., Hólm, H., Ding, E. L., Johnson, T., et al., 2012. Plasma HDL cholesterol and risk of myocardial infarction: a mendelian randomisation study. Lancet, 380(9841):572-80. 
bioRxiv preprint doi: https://doi.org/10.1101/018150; this version posted April 16, 2015. The copyright holder for this preprint (which was not certified by peer review) is the author/funder. All rights reserved. No reuse allowed without permission.

[42] Welsh, P., Polisecki, E., Robertson, M., Jahn, S., Buckley, B. M., de Craen, A. J. M., Ford, I., Jukema, J. W., Macfarlane, P. W., Packard, C. J., et al., 2010. Unraveling the directional link between adiposity and inflammation: a bidirectional Mendelian randomization approach. J Clin Endocrinol Metab, 95(1):93-9. 